Gaines, B. R. (1975). Stochastic and fuzzy logics. Electronics Letters 11, 188-189.

\title{
Stochastic and fuzzy logics
}

\author{
Brian R. Gaines \\ Department of Electrical Engineering \\ University of Essex \\ Colchester CO4 3SQ, England
}

\begin{abstract}
It is shown that it is possible to regard stochastic and fuzzy logics as being derived from two different constraints on a probability logic: statistical independence (stochastic) and logical implication (fuzzy). To contrast the merits of the two logics, some published data on a fuzzylogic controller is reanalysed using stochastic logic and it is shown that no significant difference results in the control policy.
\end{abstract}

\section{Fuzzy logic as probability logic}

The literature on fuzzy logic (Lee, 1972; Zadeh 1973) treats it very much as a new concept, distinct from that of probability logic (Rescher, 1969), even though both ascribe to events numbers in the interval $[0,1]$. This suggests that a new theoretical framework is required in which to analyse the results of practical applications of fuzzy logic. This letter is to demonstrate that fuzzy logic may be treated in terms of probability theory. This is possible because probability logic is itself not truth functional (Rescher, 1969)-the truth value of a logical expression is not uniquely determined by those of its components, and additional assumptions are necessary to determine it. It will be shown that, if a relationship of logical implication is assumed between variables, the rules of fuzzy logic apply. Conversely, if these rules do apply, there is necessarily logical implication between the variables (if they are probabilistic). This is in contrast to the more common assumption of statistical independence of variables, giving what is here termed a stochastic logic.

Fuzzy logic is an extension of Boolean logic based on Zadeh's (1965) fuzzy set theory in which the usual binary truth values ( 0 and 1$)$ are extended to include any degree of membership in the closed interval of reals $[0,1]$. The normal logic operations are defined in terms of arithmetic functions on these degrees of membership. That is, taking a capital letter as a logic variable, and the corresponding lower case letter as its degree of membership,

$$
\begin{gathered}
C=A \text { AND } B=>c=\min (a, b) \\
C=A \text { OR } B=>c=\max (a, b) \\
C=\text { NOT } B=>c=1-b
\end{gathered}
$$

These definitions coincide with the normal logic functions for the two extreme values (TRUE $=$ 1 , FALSE $=0$ ). Using this notation, but regarding, for example, $a$ as being not only a degree of membership but also the actual probability of occurrence of event $A$, one may derive the probabilistic equivalents of equations 1-3. It is assumed that the events themselves are binary in nature and either occur or do not occur. Equation 3 still applies (as usual $\neg A$ means the nonoccurrence of $A$ ); for $\mathrm{C}=\mathrm{NOT} B$,

$$
c=p(C)=p(\neg B)=1-p(B)=1-b
$$


Consider now the expressions for $a$ and $b$ in terms of the joint probabilities of the events $A$ and $B:$

$$
\begin{aligned}
& a=p(A)=p(A \wedge B)+p(A \wedge \neg B) \\
& b=p(B)=p(A \wedge B)+p(\neg A \wedge B)
\end{aligned}
$$

From these equations, given that the probabilities lie in the interval $[0,1]$, we may derive the inequalities:

$$
\begin{gathered}
0 \leq p(A \wedge B) \leq \min (a, b) \\
0 \leq a b \leq \min (a, b) \\
\max (a, b) \leq p(A \vee B) \leq 1 \\
\max (\mathrm{a}, b) \leq a+b-a b \leq 1
\end{gathered}
$$

Consider now the significance of each of the three values in these inequalities being attained:

For $C=A$ AND $B, c=p(A \wedge B)$, the conditions are:

(a) $c=0 \Leftrightarrow p(A \wedge B)=0=>A \supset \neg B$ and $B \supset \neg A$; i.e. $A$ and $B$ are mutually exclusive;

(b) $c=a b \Leftrightarrow p(A \wedge B)=p(A) p(B)$; i.e. $A$ and $B$ are statistically independent;

(c) $c=\min (a, b) \Leftrightarrow p(A \wedge \neg B)=0$ or $p(\neg A \wedge B)=0$, i.e. one of $A$ and $B$ logically implies the other.

For $\mathrm{C}=A$ OR $B, c=p(A \vee B)=1-p(\neg A \wedge \neg B)$, the conditions are:

(i) $\mathrm{c}=1 \Leftrightarrow p(\neg A \wedge \neg B)=0 \Leftrightarrow \neg A \supset B$ and $\neg B \supset A$; i.e. one of $A$ and $B$ must occur;

(ii) $c=a+b-a b \Leftrightarrow p(A \wedge B)=p(A) p(B)$; i.e. $A$ and $\mathrm{B}$ are statistically independent;

(iii) $c=\max (a, b) \Leftrightarrow p(A \wedge \neg B)=0$ or $\mathrm{p}(\neg A \wedge B)=0 \Leftrightarrow A \supset B$ or $B \supset A$; i.e. one of $A$ and $B$ logically implies the other.

It may be seen that conditions (a) and (i) are independent, and together imply that $A=\neg B$. Conditions $(b)$ and (ii) are identical and together lead to a stochastic logic in which variables are assumed to be statistically independent. Conditions (c) and (iii) are identical and together lead to a fuzzy logic in which all variables are assumed to belong to a single chain of implication. Thus, informally, the assumptions that lead to stochastic and fuzzy logics are seen to be of opposite natures, but both, arise as constraints on an underlying probability logic.

\section{Practical comparison of fuzzy and stochastic logics}

The assumptions necessary to derive a fuzzy or stochastic logic from a probability logic clearly are semantic in nature and their relative merits may be contrasted only in the context of particular applications. Mamdani and Asillian have described a fuzzy-logic controller for a steam engine in which nonnumeric linguistic statements about appropriate control strategies are converted directly into a fuzzy control policy (Assilian, 1974; Mamdani, 1974; Mamdani \& Assilian, 1975). The policy based on a collection of some 29 rules of the form 'if the pressure error is negative and big, and the change in pressure error is neither negative big nor negative medium, then the heat change should be positive and big' was found to give markedly better performance than tuned 2- and 3-term linear controllers or nonlinear adaptive algorithms. 
This application provides a clear test case where using fuzzy logic may be regarded as equivalent to postulating that, if two rules apply, the one with the lower degree of membership is subsumed by the other and may be neglected. Using stochastic logic is equivalent to postulating that the rules derive from independent considerations and both should be taken into account. One might suppose, in practice, that the actual state of affairs lies between these extremes. From a more concrete viewpoint, one might take the degrees of membership as representing the fraction of control engineers who agreed that a particular description, or rule, applied, i.e. the probability that a randomly selected engineer would agree this, and the question becomes one of whether the same engineers would choose the applicable rules, or whether the choices are distributed randomly among them. Note, however, that this is only one possible semantic interpretation of the degree of membership in this case.

As an experiment, the 29 rules for the steam-engine throttle control (Assilian, 1974; Mamdani \& Assilian, 1975) were used to generate a phase-plane portrait of the control policy using both the stochastic logic of conditions (b) and (ii) and the fuzzy logic of conditions (c) and (iii). The pressure error had been quantised at 14 levels and the change in pressure error to 13 levels, so that it was possible to compare the resulting policies in 182 cells. It was found that the two agreed completely in all except two cells. These were both on the switching line of the policy where the control action changed from positive to negative, and corresponded to the switching line being displaced by one cell. In both these cases, the degree of membership of the action selected by the fuzzy logic was only 0.2 , compared with values of 0.6 to 1.0 in the majority of cases. Thus it may be concluded that no significant difference in control policy results from combining the linguistic statements about control rules using either the 'fuzzy' or the 'stochastic' variants of probability logic.

\section{Conclusions}

The results reported in this letter highlight the significant feature of applications of fuzzy logic. This is not the precise rules of the logic itself, but rather the use of qualitative statements to produce a quantitative result. This is one role of probability theory, particularly that part of it that deals with subjective probability and decision making, two areas that in recent years have become far better formally related to the more objective formulations of probability theory (Fine, 1973). The control study cited may be regarded as a (highly successful) experiment in subjective probability applied to a real design problem. The probabilistic basis for the fuzzy logic used is an appropriate tool for the formal analysis of the controller's stability etc. The robustness of the results to radical changes in the assumptions underlying the logical calculus used is an encouraging indication of the basic robustness of the technique.

\section{Acknowledgements}

To L. Kohout for discussions of fuzzy logic and to E. H. Mamdani for access to his research data and discussion of these results.

\section{References}

Assilian, S.: Artificial intelligence in the control of real dynamic systems. $\mathrm{PhD}$ thesis, 1974, Queen Mary College, University of London.

Fine, T. L.: Theories of Probability (Academic Press, 1973). 
Lee, R. C. T.: 'Fuzzy logic and the resolution principle', Journal ACM, 1972, 14, 109-119.

Mamdani, E. H.: 'Application of fuzzy algorithms for control of simple dynamic plant', Proceedings IEE, 1974, 121, 1585-1588.

Mamdani, E. H., and Assilian, S.: 'An experiment in linguistic synthesis with a fuzzy logic controller', International Journal Man-Machine Studies, 1975, 7, 1-13.

Rescher, N.: Many-valued Logic (McGraw-Hill, 1969), chap. 2, 184-188.

Zadeh, L. A.: 'Fuzzy sets', Information \& Control, 1965, 8, 338-353.

Zadeh, L. A.: 'Outline of a new approach to the analysis of complex systems and decision processes’, IEEE Transaction Systems, Man \& Cybernetics, 1973, SMC-3, 28-44. 